\title{
A Reanalysis of Adverbs in A-not-A Questions*
}

\author{
Lili Xie \\ College of Foreign Languages and International Studies \\ Hunan University \\ Changsha, China \\ College of Foreign Languages \\ Hunan University of Science and Engineering \\ Yongzhou, China
}

\begin{abstract}
The paper proposes a pragmatic analysis of the distribution of adverbs in A-not-A questions. It argues that "most adverbs" will cause pragmatic anomaly when appearing before the A-not-A form, while temporal and locative adverbs will not. According to the ontological properties of adverbs, "most adverbs" are named as propositional adverbs and temporal and locative adverbs as possible world adverbs. Propositional adverbs ontologically are part of the proposition or event, and thus presuppose that the speaker knows something about the event. However, possible world adverbs are ontologically independent of the events and have no such presupposition. Besides these, A-not-A form induces two mutually exclusive events and presuppose an ignorant state of the speaker. The pragmatic anomaly arises from the contradictory cognitive state of being ignorant when using the A-not-A form on one hand and being non-ignorant when using propositional adverbs and a specific verb on the other hand. However, possible world adverbs are ontologically independent of the events and thus compatible with the ignorance presupposition of the A-not-A form. The analysis predicts the distributive pattern of speech act adverbs in A-not-A questions.
\end{abstract}

Keywords-the distribution of adverbs in A-not-A question; pragmatic anomaly

\section{INTRODUCTION}

The distribution of adverbs in A-not-A questions (abbrev. as ANAQ) in Mandarin Chinse has been widely discussed (1-7). It's said that temporal and locative adverbs can appear before the A-not-A form (abbrev. as ANA form), while most adverbs, i.e., those expressing manner, degree, aspect, frequency, modal and so on cannot, as shown by examples $(1-2)$ and (3-7) respectively.

(1) Lisi mingtian qu-bu-qu?

Lisi tomorrow go-not-go

Is Lisi going-or-not-going tomorrow?

(2) Lisi zai jia li zuo-bu-zuo gongke?

Lisi at home do-not-do homework

Does Lisi do-homework-or-not-do-homework at home?

*This work is supported by the Provincial Social Science Foundation (No.13YBA402) and the Natural Science Youth foundation of Hunan Province (No. 2018JJ3192) in Hunan China.
(3) *Lisi xiaoxin de kan-bu-kan shu?

Lisi carefully read-not-read book

Is Lisi carefully reading-books-or-not-reading books?

(4) *Lisi hen cong-bu-congming?

Lisi very clever-not-clever

Is Lisi very clever-or-not-clever?

(5) *Lisi turan hui-mei-huijia?

Lisi suddenly go-not-go home?

Did Lisi suddenly go-home-or-not-go-home?

(6) *Lisi jingchang qu-bu-qu Beijing?

Lisi often dance-not-dance

Does Lisi often go-or-not-go to Beijing?

(7) *Lisi yiding qu-bu-qu?

Lisi definitely go-not-go?

Is Lisi definitely going-or-not-going?

The most influential view given by the former researchers attributes this distributive pattern to a blocking effect or a semantic interpretation crash. For example, Law [4] proposes the constraint (8). It says that ANA morpheme has to LF move to sentence initial position to get interpreted; if the adverb (like adverbs in sentences (3-7)) in between is a potential binder, it will block the movement and thus cause semantic interpretation crash; otherwise (like adverbs in sentences (1-2)), it won't.

(8)

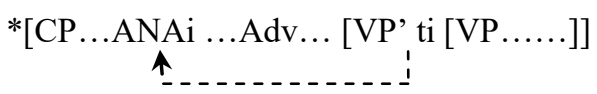

Attributing the ungrammaticality of sentences like (3-7) to the semantic interpretation crash caused by the constraint (8) equals to admitting the intended meaning expressed by these sentences are logically reasonable. The problem is that the intended meaning expressed by sentences like (3-7) are intuitively absurd, as shown by the odd English translations. For instance, if the speaker of (3) did not know whether the reading event is in progress, how could he know its manner property? Or vice versa, since the speaker knows the manner 
of the reading event, he should have known that this event is in progress. Under this case, how can he use the ANA form, which expresses his doubting whether the action is in progress? The same problem exists in (4): if the speaker of (4) knows Lisi possesses a high degree of along the line of cleverness, he should know whether this thing is being clever or not being clever. Therefore, this cognitive state is also contradictory with the ANA form, which expresses his ignorance state. Thus we take the fact that the speaker of sentences like (3) and (4) knows the manner or degree but don't know the action or the thing bearing the correspondent property is cognitively unreasonable. However, in line with Law's constraint (8), these meanings are logically acceptable, which obviously disobeys our intuition. Therefore, we hold that any analysis on the way of Law's constraint (8) is not on the right track.

This paper proposes a pragmatic analysis of the distribution of adverbs in A-not-A questions. It argues that "most adverbs" will cause pragmatic anomaly when appearing before the A-not-A form, while temporal and locative adverbs will not. According to the ontological properties of adverbs, "most adverbs" are named as propositional adverbs and temporal and locative adverbs as possible world adverbs. Propositional adverbs ontologically are part of the proposition or event, and thus presuppose that the speaker knows something about the event. However, possible world adverbs are ontologically independent of the events and have no such presupposition. Besides these, Anot-A form induces two mutually exclusive events and presuppose an ignorant state of the speaker. The pragmatic anomaly arises from the contradictory cognitive state of being ignorant when using the A-not-A form on one hand and being non-ignorant when using propositional adverbs and a specific verb on the other hand. However, possible world adverbs are ontologically independent of the events and thus compatible with the ignorance presupposition of the A-not-A form. The analysis predicts the distributive pattern of speech act adverbs in A-not-A questions.

Section two discusses the semantic differences between types of adverbs, including speech act adverbs, which were not discussed by Law. Section three discusses the contradictory cognitive state caused by the contradiction between using the ANA form to seek information and the content presupposed by "most adverbs" and the two mutually exclusive events introduced by the ANA form. And on the basis of Oshima [8], we attribute this phenomenon as a pragmatic anomaly.

\section{TYPES OF ADVERBS}

\section{A. Law's Division of Adverbs}

In fact, Law's proposal of the constraint (8) was made on the basis of the distribution and the semantic property of the adverbs in ANAQ. He argues that "most adverbs" in sentences like (3-7) has a close semantic relation such as entailment with the event or state expressed by the predicate, while locative and temporal adverbs are world and time coordinates in model-theoretic theory. Thus he calls the former predicate-related adverbs, and the latter not-predicate related adverbs. According to their semantic relation with the predicate, Law argues that predicated-related adverbs are potential binders and not-predicate-related adverbs are not, thus explaining the distribution of adverbs in ANAQ with the constraint (8).

Obviously, terms of (not-) predicate-related adverbs in fact cannot distinguish "most adverbs" from locative and temporal adverbs, and may even cause a mess. On the one hand, locative and temporal adverbs are used to describe the temporal and locative property of the events or state, they are thus of course predicate related like predicate-related adverbs. On the other hand, adverbs like "definitely" do not entail the event or state expressed by the predicate, while locative and temporal adverbs like manner adverbs do. These semantic relations are totally contradictory with the diagnosis given by Law. Therefore, we think to use semantic relations like entailment as Law cannot explain the distribution of adverbs in ANAQ.

\section{B. Ontological Distinctions Among Adverbs}

However, we do agree that the adverbs divided by their distribution pattern in ANAQ have semantic distinctions, because they are indeed ontologically different. We can put it simply with the help of possible world semantic theory, which define the truth of propositions with the function $p(w)$ with $\mathrm{p}$ standing for the proposition and $\mathrm{w}$ the possible world [9]. With this, it's easy to distinguish the two types of adverbs ontologically, because locative and temporal adverbs are used to describe the possible worlds, while "most adverbs" are used to describe the proposition.

Usually, if there is no possible world description in the sentence, the proposition will be checked in the actual world by default. For instance, to check the truth of the proposition expressed by (9a) is to define whether there is a detective living in 221B Baker Street in the actual world. However, to check the truth of $(9 b)$ requires to define whether there is a detective living in 221B Baker Street in the world of Sherlock Holmes that is related with the actual world.

\section{(9) a. A detective lives at 221B Baker Street.}

b. In the world of Sherlock Holmes, a detective lives at 221B Baker Street.

According to this distinction, we'd like to replace the terms of predicate-related adverbs and not-predicated-related adverbs with propositional adverbs and possible world adverbs. However, besides these two, there is a third type of adverbs, which is used to describe neither $\mathrm{p}$ nor w. they are used to describe speech acts, such as intentionally and reluctantly.

From Law's analysis, we know that propositional adverbs and possible world adverbs contribute to the truth of a proposition, and the former is incompatible with ANA form, while the latter is. However, for speech act adverbs, they are either compatible or incompatible with ANA form, as shown in (10a-b) and (11a-b).

$$
\begin{aligned}
& \text { (10) a. } \text { Lisi guyi qu-bu-qu ? } \\
& \text { Lisi intentionally go-not-go }
\end{aligned}
$$


Is Lisi intentionally going-or-not-going?

b. *Lisi xinbuzaiyan-de kan-bu-kan shu

Lisi absent-minded-ly read-not-read books

Is Lisi absent-mindedly reading-or-not-reading

books?

(11) a. Lisi daodi qu-bu-qu?

Lisi really go-not-go

Is Lisi really going?

b. Lisi jiujing qu-bu-qu?

Lisi really go-not-go

Is Lisi really going?

The distribution of speech act adverbs in ANAQ above shows that the classification of adverbs alone cannot give a satisfactory answer to the distribution of adverbs in ANAQ. Next section we will follow our intuition in the introduction and to give a preliminary analysis.

\section{PRESUPPOSITION FAILURE AND PRAGMATIC ANOMALY}

In the introduction part, we mentioned that adverbs before ANA form may put the speaker in a cognitively contradictory position. Next we will give a detailed analysis to explain this intuition and proposes a pragmatic analysis for the distribution of adverbs in ANAQ.

The basic function of questions is to seek information. For ANAQ, what the speaker wants to do is to get which one of the two mutually exclusive events is true from an ignorant information state. Our basic idea is that "most adverbs" before ANA form presuppose the speaker is not in an ignorant state. That is, the speaker actually has known the answer before asking the ANAQ, which will disable the basic function of questions. We attribute this case to a presupposition failure. Temporal and locative adverbs before ANA form do not have such a presupposition and thus are compatible with the ignorance presupposition of ANA form.

\section{A. Presupposition of Adverbs}

In event semantics, a predicate introduces an event, and adverbs pose semantic constraints on the event argument. For instance, the sentence (12) and (14) are given logical forms along the lines of (13) and (15), where the predicate introduces an event variable e, and the adverbs are used to constrain the event.

(12) a. Lisi jingchang qu beijing.

Lisi often go Beijing

Lisi often goes to Beijing.

(13) $\exists \mathrm{e}[\mathrm{go}(\mathrm{e}$, Lisi, Beijing) $\wedge$ often (e)]

(14) a. Lisi mingtian qu.

Lisi tomorrow go
Lisi is going tomorrow.

(15) $\exists \mathrm{e}[\mathrm{go}(\mathrm{e}, \mathrm{Lisi}) \wedge$ tomorrow (e)]

The ANAQ expressed in (1-7) all consists of an adverb and an ANA form. The adverb and the ANA predicate belong to the common ground, and the ANA form expresses the inquisitive content. Take sentence (1) and (6) for example. Their logical forms are given along the lines of those in (16) and (17). The ANA form in the two kinds of ANAQ contributes the same meaning of introducing two mutually exclusive events. However, we argue that propositional adverbs and possible world adverbs have different presuppositions when used together with the ANA form according to their ontological differences.

(16) $\exists \mathrm{e}[\mathrm{e} \in\{\mathrm{go}(\mathrm{e} 1, \mathrm{Lisi}), \neg \mathrm{go}(\mathrm{e} 2, \mathrm{Lisi})\} \wedge$ tomorrow (e)] (e)]

(17) $\exists \mathrm{e}[\mathrm{e} \in\{$ go(e1, Lisi, BJ), $\neg \mathrm{go}(\mathrm{e} 2, \mathrm{Lisi}, \mathrm{BJ})\} \wedge$ often

For the ontological differences, we argue that the adverbial property expressed by propositional adverbs is part of the event, while that expressed by possible world adverbs is not. For the ANA form, it introduces an event variable and expresses two mutually exclusive events as the domain of the variable, such as being clever and not being clever, or going to Beijing and not going to Beijing.

Therefore, for ANAQ with adverbs, there are actually two aspects presupposed: one is the content of the adverbs, and the other is the concrete content of the events expressed by the predicate. Since propositional adverbs contribute part of the events, we argue that knowing propositional adverbs suffices to know the correspondent event.

Things are different for locative and temporal adverbs. From the function $\mathrm{p}(\mathrm{w})$ defining the truth of propositions, we know that any proposition should be placed in a possible world to check its truth value. This means that knowing a possible world concerns nothing with the proposition or the event per se.

Based on these, we propose propositional adverbs and possible world adverbs in ANAQ have different presuppositions (18):

(18) Propositional adverbs before the ANA form presuppose that the speaker knows something about the event, while possible world adverbs in ANAQ does not have such a presupposition.

\section{B. The Presupposition Failure of $A N A Q$}

A-not-A questions semantically is a realization of polar questions and pragmatically functions as neutral questions. It divides the possible worlds into two opposite sets, that is, $\{p$, $\neg \mathrm{p}$ \}. That is, when an ANA form combines with a verb, it induces two mutually exclusive events and presuppose speaker's ignorance of the truth of the two mutually exclusive events.

Since the basic function of ANAQ is to seek information from an ignorant information state, the presupposed content in the ANAQ with preposed propositional adverbs will 
encounter a contradictory cognitive state: the ignorant speaker has already known something about the event. This knowledge state combining the two mutually exclusive information states further renders the answer to the ANAQ. That is to say, the speaker has already known the answer before asking the ANAQ. Such a contradictory situation will only occur with an irrational mind.

However, since temporal and locative adverbs before ANA form do not have such a presupposition, the requirement of using the ANAQ to seek information can be satisfied. Thus they will not affect the grammaticality of ANAQ.

Based on this, we propose that the ungrammaticality of ANAQ with preposed propositional adverbs is caused by a presupposition failure. The ANA form in the question requires an ignorant inquisitive cognitive state of the speaker. That is, the speaker is expecting an answer. However, the answer has already been presupposed by the combination of propositional adverbs and the ANA form. That is, the inquisitive content presupposed by the ANA form cannot be satisfied.

The special point is that this kind of presupposition failure can never get repaired, since it is not contigent. Oshima [8] characterize this as pragmatic anomaly, since it behaves like contradiction (e.g. it is raining and it is not raining.) or oxymoron (e.g. a round square).

\section{Speech Act Adverbs in $A N A Q$}

The same happens to the speech act adverbs. Adverbs like "daodi" and "jiujing" in mandarin Chinese are inquisitive in nature, and cannot presuppose that the speaker knows the answer to the question. Therefore, they are compatible with the ignorant cognitive state expressed by ANA form.

Adverbs like "guyi" and "xinbuzaiyan-de" although are not propositional adverbs, they express the speaker's evaluation and attitude towards an event. However, how can a speaker give an evaluation or attitude towards an event without knowing anything about the event itself?

As mentioned above, the specialty of ANAQ is that it has provided two mutually exclusive events as the domain of the variant. The ANA form will put the concrete content of the events expressed by the predicate into the common ground. Therefore, just like the case of propositional adverbs, the speaker's knowledge contained in adverbs like "guyi" and "xinbuzaiyan-de" also suffices the speaker to assign the event variable a value, which results the same pattern exactly like propositional adverbs in ANAQ.

\section{CONCLUSION}

The paper gives a pragmatic analysis of the distribution of adverbs in ANAQ. We get that the (un)grammaticality arises from the fact that there is (no) contradiction between what is known and what information is to be sought: if the information is to be sought is already in the common ground, to use an ANA form in this situation will cause pragmatic anomaly, i.e., the presupposition failure which cannot be get repaired. And the direct consequence is that the speaker has already known the answer before using the A-not-A question to seek information, which will disable the basic function of information seeking of questions and thus renders them trivial as questions.

Pragmatic anomaly is widely used to explain grammatical phenomena like islands effect, NPI and so on. The grammaticality of ANAQ is constrained not only by adverbs, but also by islands and quantificational expressions. However, although these factors are completely different from adverbs grammatically, it is possible to unify them with adverbs along the line of pragmatic analysis, based on the analysis in this paper.

\section{ACKNOWLEDGMENT}

I would like to thank Chuansheng He and Jiahui Yang for inspiring this paper and helping with various aspects of it.

\section{REFERENCES}

[1] Ernst, Thomas. Conditions on Chinese A-not-A Questions, Journal of Chinese Linguistics (1994)3: 241-264.

[2] Gasde, Hans-Dieter. Yes/no questions and a-not-a questions in Chinese revisited. Linguistics, 2004.

[3] P. Law, Adverbs in A-not-A questions in Mandarin Chinese [J] Journal of East Asian Linguistics (2006) 2: 97-136.

[4] Tseng, Wen-Hise. A Post-Syntactic Approach to the A-not-A Questions. UST Working Papers in Linguistics, National Tsing Hua University, Hsinchu, 2009: 107-139.

[5] von Prince, Kilu. Predication and information structure in Mandarin Chinese, Journal of East Asian Linguist (2012)21:329-366.

[6] Xu Liejiong. Are A-not-A Questions Alternative Questions or WhQuestions? Breaking Down the Barriers (2013)1:305-309.

[7] Jin, Dawei. The Semantics-Pragmatics Interface and Island Constraints in Chinese, PhD Dissertation, State University of New York, 2016.

[8] Oshima DY. On factive islands: pragmatic anomaly vs. pragmatic infelicity. In Proceedings of the Annual Conference of the Japanese Society for Artificial Intelligence, ed. T Washio, 2006: 147-61. Berlin: Springer.

[9] von Fintel, Kaivon and Heim, Irene. Lecture Notes in Intensional Semantics, MIT, 2011 